Da nun auch bei der Behandlung mit schwefelichter Säure dic Ausbeute an weissem Talg so geringe ist, so komme ich au? meinen schon oben gegebenen Vorschlag zurück. Man tröpfle Thranschmalz ab und benutze das 0 el wie gewöhnlich zum Brenuen; den abgetröpfelten Thranschmalz reinige man durch Schmelzen und Durchseihen; und wende auch ihn als Beleuchtungsmittel an. Einen andern Theil des abgetröpfelten Schmalzes möge man mit Schwefelsäure reinigen und zum Seifensieden anwenden; auch allenfalls einen Antheil desselben stcif pressen, und mit Talg zu Talglichtern verbrauchen.

II.

Ueber die Stärke.

Auszug einer der Akademie der Wissenscliaften am 4. Mal 1835 vorgelesenen Abhandlung

ron

G U ́́ BI N - VA R B Y.

(Institut Nr. 105.)

Die Abhandlung zerfält in drei Theile, in welchen der Verfasser nach einander folgende 3 Gegenstände abhandelt: 1) die Wirkung der Diastase nuf das Kartoffelstärkemehl bei verschiedenen Temperaturen. 2) den durch diese Wirkung erzengten Zucker, verglichen mit dem durch Schwefelsäure erzeugten. 3) die gummige Materie, welche gleichfalls durch die Wirkung der Diastase auf das Stärkemehl entsteht.

\title{
1. Theil.
}

Wirkung der Diastase auf das Kartoffelstärkemehl bei verschiedenen Temperaturen.

Der Verf. suchte zuverlässig zu bestimmen, wie viel Zeit und eine wie grosse Menge Diastase erforderlich sei, um ein gegebenes Gewicht Stärkemehl in Zucker und Gummi bei einer bekannteu Temperatur und mit einer bekannten Menge Wasser zu verwandeln. Das Resultat seiner Versuche war folgendes :

1) Bei einer Temperatur zwischen $70-75^{\circ}$ gaben 100 Th. Stärkemehl mit Inbegriff der Tegumente, mit $1000 \mathrm{Th}$. 
Wasser und 1,7 gr. Diastase nach Verlauf von 6 Stunden 17,58 Theile Zucker;

2) Zwischen 60 und 650 gaben 100 Th. Stirke in Kleister verwandelt mit ungefihh $39 \mathrm{Th}$. Wasser und dann mit 6,13 gr. Diastase gemengt, die in $40 \mathrm{Th}$. Wasser aufüelöst war, nach Verlauf einer Stunde 86,91 Theile Zucker;

3) Ein Kleister, welcher 100 Th. Stirke aut 1393 Wasser enthieit mit 12,25 Th. Diastase, die in 367 Th. kaltem Wasser gelöst war, gab bei 24stündiger Behandlung in einer Temperatur von $20078,64 \mathrm{Th}$. Zucker.

Dieses Resultat, sagt der VR., scheint mir von hoher Wichtigkeit, weil man nicht nur Brennmaterial bei Verwandlung des Stir kemehls in Zucker sparen laun, sondern auch einen grossen Theil der Kosten, welche die Destillation der schwachen alkoholhaltigen Flüssigkeiten verursacht, die man bei dem gewölnlichen Verfahlen des Branntweinbrennens aus Kartoffeln erbält. Es ist nümlich behannt, dass man nach Verwandlung des Zuckers in Stärkemehl bei einer Temperatur zwischen $60-65^{\circ}$ genöthigt ist, der zuckerhaltigen Flüssigkeit ein gleiches Volumen kaites Wasser zuzusetzen, um ihre Temperatur auf $15-30^{\circ}$ herabzustimmen, bei welcher man die Grihhung beginnen lässt. Man erhiilt ïuf diese Weise sehr wenig alkoholhaltige Flüssigkeiten, die man mit grossen Kosten destillirt. Auf den zuletzt genaanten Versuch gestützt wird man das kalte Wasser, das der zuckerhaltigen Flüssigkeit zugefügt wird, unmittelbar mit dem bei $20-21^{\circ}$ bereiteten Kleister mischen und dadurch das Product vermehren.

4) Der vorhergehende Versuch, bei der Temperatur des schmel\%enden Eises wiederholt, gab nach Verlauf von 2 Stunden 11,82 Th. Zucker.

5) Bei einer Temperatur zwischen 12 und $5^{\circ}$ machte die Diastase den Kleister füssig, aber es fand keine Zackerbildung statt.

Da die Art der Einwirkung der Diastase mit dem Kleister gänzlich unbekannt ist, so untersuchte der Verf., ob während derselben eine Entwickelung oder Absorption von Gas statt fïnde. Er hat indessen nichts der Art wahrnehmen können und fand, duss die Reaction ebeu so gut an der Luft als im luftleeren Raume vor sich geht. 
Ferner untersuchte er die Wirkung des Wassers auf die Stärke bei verschiedenen Temperaturen, um sie mit der der Diastase unter denselben Umstïnden zu vergleichen. Zu diesem $Z \mathbf{Z w e}-$ cke untersuchte er unter dem Mikroskope mit $H$. Turpin die Stärkemehlkügelchen, welche verschiedenen Einwirkungen unterworfen worden waren. Er giebt über diese Versuche folgenden Bericht:

1) Stärkemehl im natürlichen Zustande. Die kleinsten Körner sind sphärisch, die grössten länglich, oder am häufigsten dreiseitig mit abgerundeten Kanten. Im Mittelpuncte der sphärischen Körner, oder in einer der Ecken der dreikantigen, unterscheidet man den Nabelpunct, vermittelst dessen der organisirto Körper an der Wandung der Zelle anhing. Rings um denselben finden sich concentrische Zonen, ähnlich denen, welche der Querschnitt des Stammes der Dikotyledonen zeigt. Die Kügelchen sind glatt an ihrer Oberfläche, durchscheinend, farbenlos und sehr schwach perlmutterglïnzend.*) Man bemerkt daran keine Spur von körniger Textur; mit Wasser behandelt treten sie aı dasselbe keine Spur einer jodbläuenden Sabstanz ab, dasselbe verhält sich nachher ganz neutral gegen Pflanzenfarben. Bisweilen finden sich die einzelnen Kürnchen zu $z$ weien und dreien verwaclisen, wobei jedoch die Nabelpuncte immer nach aussen gekehrt sind.

2) Stärkemehl, welches eine Stunde lang der Wirkung des Wassers bei verschiedenen Temperaturen (3 Th. Stärke auf 50 Wasser) mit 2 Th. Diastase oder ohne solche ausgesetzt worden ist.

a) Die Temperatur 50 - $53^{\circ}$. Die Körnchen haben die nämliche Form wie vorher, man mag Diastase anwenden oder nicht. Ohne Diastase giebt die filtrirte, durchsichtige und beinahe zur Trockne gebrachte Flüssigkeit nicht die mindeste Fürbung mit Jod. Mit Diastase hat die durchsich-

*) Die hier in sehr abgekürztem Ausznge milgetheilten mikroskopischen Beobachtungen stimmen beinahe genall mit den sehr sor gfiiltigen Cutersuchungen der Stärkekörnchen von Fritzsche (Pogro. Aun. Bd. 32. 129) iiberein, nur ist die Bedeutung der beobachtetell Erscheinungen von dem $\mathbf{V f}$, anders und wahrscheinlich minder richti; aufgefisst worden.

d. $\boldsymbol{H}$. 
tige Flüssigkeit ebenfalls keine Wirkung auf Jod; Bierhere bewirkt darin bei $25^{\circ}$ keine Entwickelung von Kohlensiure.

b) Temperatur von 54 - 550. Ohne Diastase scheint eine Anzahl der blasenartigen Kügelchen, etwa 1 auf 200, von dem Nabelpuncte aus aufgerissen zu sein. Die filtrirte Flüssigkeit giebt nach dew Eindunsten eine kaum merkliche Färbung mit Jod. Mit Diastase erhält man ein ähnliches Resultat, die filtrirte Flüssigkeit gab mit Bierhefe einige Blasen von Kohlensäure, die von Spuren von Zucker abzuhängen schienen.

c) Temperatur von $59-60^{\circ}$. Obne Diastase sieht man sehr viele zerrissene Kügelchen. Die filtrirte, durchsichtige Wlüssigkeit bläuet sich stark mit Jod. Mit Diastase, gleiche Veränderung der Kügelchen, die klare filtrirte Flüssigkeit gährte mit Bierhefe.

d) Temperatur von $60-61^{\circ}$. Ohne Diastase sind eine eine grosse Menge von Kürnern zerplatzt oder zerrissen, die filtrirte Flüssigkeit färbt sich stark mit Jod. Mit Diastase gleicher Zustand der Kügelchen, die filtrirte Flüssigkeit nimmt keine Färbung mit Jod an und gälurt stärker als die in c) eriballene.

e) Temperatur von $61-620$. Ohne Diastase: fast alle Küruer sind zerrissen und zertheilt, die filtrirte Flüssigkeit färbt sich intensiv blau mit Jod. Beobachtet man das Stärkemehl in der Röhre, worin es erhitzt wird, so sieht man es allmählich aufschwellen, es bildet mit Wasser einen so consistenten Kleister, dass er am Boden des Gefïmes bleibt, wenu man dasselbe umkehrt. Mit Diastase: die Körner fast alle geplatzt aber nicht zertheilt wio ohne Diastase. Die filtrirte Flüssigkeit giebt keine Fürbung mit Jod, sie hat die geistige Gährung erlitten.

P) Temperatur zwischen $63-64^{\circ}$. Gleiche Resultate wie bei e).

g) Temperatur von $62-64^{\circ}$. Ohne Diastase: alle Isörner sind in dünne Hïutchen verwandelt. Die filtrirte Flüssigkeit fürbt sich intensiy blau mit Jod. Mit Diastase erscheinen die Körner blos an dem einen Ende aufgerissen, die filtrirte Flüssigkeit gïhrt stark mit Hefe.

h) Temperatur ron $6.1-65^{\circ}$. Ohne Diastase siebt man 
nur äusserst düme durchsichtige Häutchen, dje Flüssigkeit färbt sich stark mit Jod. Mit Diastase ist alles wie bei g).

H. Guérin schliesst den ersten Theil seiner Abhandlung mit folgenden Worten.

1) Das Wasser bewirkt unter Beihülfe der Wärme das Zerplatzen der Stärkekürner von $54^{\circ}$ an, und die Diastase in Ueberschuss angewandt, weit enternt, diese Wirkung zu begünstigen, hindert vielmehr unter gewissen Umstïnden das vollständige Zerreissen derselben.

2) Die Diastase hat durchaus keine Wirkung auf die nock nicht zerplatzten Stärkemehlkörner, es macht blos den Stärkelleister flüssig und verwandelt ilhn in Zucler.

3) Die Diastase wirlit nicht durch dic Hüllen hindurch, sie bewirkt keinesweges das Zerplatzen derselben, durch eine Art von Endosmose, wie die Herrn Pay en und Dutroch et glauben.

4) Im Acte des Keimens bildet also die viastase keinesweges den innern und für unauflöslich gehaltenen Theil der Körner in zwei neue und lösliche Substanzen um.

\section{Theil.}

I. Zucker aus stärkemehl mittelst Diastase bereitet.

In seiner letzten Abhandlung über das stärkemehl und die Diastase *) behauptet $\mathbf{P}$ ay en, dieser Zucker sei nicht krystallisirbar und erstarre nicht zu lester Masse, wie der mit Stirkemehl and Schwefelsiture bereitete, er sei unlüslich in Alkohol von $95^{0}$ bis zum wasserereien Zustande, und er wandle sich unter Einfluss der Hefe, des Wassers und der geeigneten Temperatur vollstïndig in Kohlensäure und Alkohol um. Die in diesem zweiten Theile angeführten Versuche widersprechea diesen Behnuptangen. H. Guérin erinnert zuvörderst daran, dass Dubrunfaut diesen Zacker zuerst in Krystallen gesehen habe, in einem mil gekeimter Gerste und Stailse der freiwilligen Verdunstung überlassenen syrup. H. Guérin hat den Gegenstand weiter verfolgt, und ist dabei zu fulgenden Resultaten gekominen:

*) Dieses Jourual Bd. 4. s. 288 . 
A. Eigenschaften dieses Zuckers. Er ist weiss, geruchlos, hart, knirscht zwisclsen den Zühnen, besitzt einen frischen und in Vergleich mit Rohrzucker wenig süssen Geschmack. Er krystallisirt in Gestalt blumenkohlartiger Massen, und in Prismen mit rbombuidalen Flächen. Sein Epecifisches Gewicht in Olivenöl bestimmt, und auf das des Wassers reducirt, ist 1,3861, folglich geringer das des Rohrruckers, welches 1,6065 beträgt. Bis zu 600 erhitat erweicht er sich, dei $70^{\circ}$ wird er teigig, bei $90^{\circ}$ syrupartig. Wird er eine stunde lang bei $100^{\circ}$ erhit $\%$, so verliert er 9,80 auf 100 seines Gewichts. Verschiedene Versuche zcigten, dass diese Temperatur am geeignetsten ist, um ihm sein Krystallwasser ohne Zersetzung zu entziehen. Löst man ihn in siedendem Alkohol von 950 auf, nachdem man ihm durch Erhitzen sein Krystallwasser entzogen hat, und überlässt die Flüssigkeit der Ruhe, so setht er sich bein Erkaiten in farbenlosen, blumenkohlartigen Krystallen ab. Er ist in allen Verhältnissen in siedendem Wasser löslich, während $100 \mathrm{Th}$. Wasser von 23,50 nur 63,25 Th. desselben auflüsen. Der Alkohol löst davon un so mehr auf, je weniger concentrirt er ist. In Olivenöl ist er in der Kälte unauflöslich. Den Alkohol hält er sehr hartnächig zurück. Seine Zusammensetzuug ist;

$$
\mathrm{C}_{13} \quad \mathrm{H}_{28} \quad \mathrm{O}_{14}
$$

also gleich der des Rübenzuckers. Der Stärkezucker wird demuach reprïsentirt durch krystallisirten Rohr\%ucker plus 3 Atomen Wasser. Die Versuche des Verfassers, dem Zucker diese 3 Atome Wasser zu entziehen, waren fruchtlos,

B. Bereitung. Man zerrührt 100 Th. Stärke in 400 Th. kaltem Wasser, giesst das Gemenge in 200 Th. siedendes Wasser und rüht rasch um. Es entsteht ein wenig consistenter Kleister, dessen Temperatur man auf $65^{\circ}$ erniedrigt. Man fügt demselben sodann 2 Th. Diastase, in 20 Th. kaltem Wasser gelöst, zn und rührt wohl um. Die Temperatur wird sodann $21 / 2$ Stunde lang xwischen 60 - 650 erhalten, worauf die Flüssigkeit bei $60^{\circ}$ so rasch als möglich, am besten im luttleeren Raume, so weit ab- 
gedampft wird, dass sie $3^{0}$ am Beaumè'schen Aräometer zeigt. Wird das Product sodann in flachen Gefässen der Luft ausgesetzt, so bildet es nach Verlauf einiger Tage eine syrupartige Masse, in welcher man hie und da körnige Krystalle unterscheidet. Diese Masse wird mit Alkohol von $95 \%$ bei einer Temperatur von $74^{\circ}$ behandelt, man lässt die Flüssigkeit sodann bei abgehaltener Luft erkalten und giesst sie durch ein Filterpapier. Die filtrirte Flüssigkeit wirdim Wasserbade bis zur Syrupsconsistenz eingedampft. Man bringt den Syrup unter die Glocke der Luftpumpe, wo er dann sehr bald krystallisirt. Die erhaltenen Krystalle werden zwischen doppeltem Fliesspapier ausgepresst, bis sie keine gefürbte Flüssigkeit mehr von sich geben. Darauf werden sie von Neuem mit Alkohol behandelt. Die neuen Krystalle werden in dem Vierfachen ihres Gewichts Wasser von $\mathbf{6 5}^{\circ}$ aufgelüst, man setzt der Aufösung 1/10 gereinigter thierischer Kohle zu und unterhäit die Temperatur unter bestïndigem Umrühren eine halbe Stunde lang. Die filtrirte warme Flüssigkeit wird im luftlecren Raume zur Krystallisation abgedampft. Um die Krystalle von dem letzten Reste des anbängenden Alkohols zu befreien, den sie sehr fest halten, löst man sie endlich nochmals in dem Vierfachen ihres Gewichtes Wasser von $65^{0}$ auf und lisst sie von Nenem krystallisiren, was man zuletzt noch einmal wiederholen kann.

II. Zucker aus Stärkemehl mit Schwefelsïure bereitet.

Dieser Zucker ist schon von Th. v. Saussure studirt worden. Sein specifisches Gewicht ist 1,391, seine Krystallform und seine Zusammensetzung stimmen mit dem vorigen überein, und es gilt von ihm überhaupt Alles, was von dem vorigen angegeben worden ist. Indessen gelang es H. Guérin, denselben ganz frei von jeder gelblichen Färbung, vollkommener weiss als den schönsten Rohrzucker, nach folgendem Verfahren zu erhalten.

Nachdem man den Zucker auf die gewöhnliche Weise bereitet hat, presst man die noch feuchten Krystalle zwischen Fliesspapier so lange aus, als dieses noch gefärbt wird. Man löst dann das product in 4 Theilen Wasser von $65^{\circ}$ auf, rührt die Auflösung $1 / 2$ Stunde lang mit $1 / 10$ ihres Gewichts gerei- 
nigter thierischer Kohle um, und bringt das Ganze auf ein Filter. Dic filtrirte Flüssigkeit wird im luftleeren Raume zur 'Trockniss abgeraucht. Die schwach gelblichen Krystalle werden von Neiem aufgelöst und mit thierischer Kohle behandelt, und die Aullüsung in luftleeren Raune abgedamplt. Wean sie dicke Syrupsconsistenz zeigt, vollendet mau die Krystallisation an freicr Luft bei gewöhnlicher Temperatur. Das Auspressen zwischen Papier hat den Zweck, den feuchten Krystallen eine syrupartige Substanz zu entziehen, die ihrer Entfärbung linderlich zu sein scheint.

\section{3. 'Theil.}

Gummiartige Substanz, welche durch die Wirkung der Diastase auf den stirkekleister erzengt wird.

A. Zitgenschaften. Die Substanz ist weiss, geschmacklos, geruchlos, durchsichtig in dünnen Platten, nach dem Austrocknen ist sie zerreiblich, der Bruch glasig. Sie röthet kaum schwach gehlitietes Lackmuspapier. Mit Jod gicbt sie keine blaue Firbung. Sie erweicht nicht bei $100^{\circ}$, zwischen $125-130^{\circ}$ giebt sie Wasser aus und nimmt eine gelbliche Firbung so wic einen Geruch nach geröstetem Brode an. Zwischen $\mathbf{1 9 5}-\mathbf{2 0 0 0}$ wird sie rothbraun, bei $235^{0}$ schmilæt sie, blaht sich betriehtlich aut, nimmt eine gelbbraune Farbe an und entwickelt Kohlensïure, Kohlenwasserstofi, Essigsiure u. s. w. An trockier Luft ist sie unverinderlich. Sie ist unlöslich in absolutem Alkohol, und Schwefelither, löst sich in kleiner Menge in Alkohol vou $88^{\circ}$ auf und ist sehr lüslich in kalten und heissem Wasser. Sie giihrt nicht mit Bierhefe und Wasser. Mit salpetersäure behamlelt giebt sie keine Schleimsätre.

Die Diastase verwaindelt, selbst im Leberschusse angewandt, die gummige Substanz, welche sich in der Mutterlange des Stirkezuckers findet, nicht in Zucker, wenu sie aber isolirt ist, wird sie dadurch fast vollstinding in Zucker verwandeit. Diese Thatsache, welche Payea in seiner letzten Ablandling läugnet, wird durch folgenden Versuch bewiesen.

Es wurden 5 gr. der gummigen Substan mit 0,5 gr. Diastase in $60^{\circ}$ Wasser von gewöhnlicher Temperatur auf- 
gelöst, die Aulösung fünf Stunden lang bei $60-6$ ŏ$^{\circ}$ erhalten, woranf sie mit $1 \mathrm{gr}$. Hefe versetzt wurde. Es entwickelte sich ein Volumen Kohlensäure, welches 3,0 29668 gr. Zucker entsprach. Hiernach geben $100 \mathrm{Th}$. der Substanz 61,459 Th. Zucker. Als man diesen von der grummigen Substanz isolirte und den Versuch von Neuem begann, wurde die letztere fast vollständig bis auf $1 \frac{1}{2}$ p. C. in Zucker verwandelt.

Es ergiebt sich hieraus, dass die sogenannte gummige Substanz kein wahres Gummi ist.

Bemerkung. Wenn man bei dem angegebenen Verfahren zur Bereitung des Stürkezuckers, mit Hülfe der Diastase, einen Rückstand erhalten hat, der zum grossen Theile aus gummiger Substanz und etwas Zucker besteht, so wird dieser letztere durch Alkohol von 95 p. C., bei der Temperatur von $75^{\circ}$ ausgezogen. Man löst dann die Substanz in dem Achtfachen ihres Gewichts Wasser von $75^{\circ}$ auf, setzt 1/20 thierische Kohle hin$z u$, womit man eine halbe Stunde lang umrührt, worauf das Ganze auf ein Papierfilter gebracht wird. Die filtrirte farblose Flüssigkeit wird im luftleeren Raume eingetrocknet.

Schliesslich bemerkt Herr Guérin, wie er in Lidfe seiner Untersuchungen gefunden habe, dass die Diastaso keine Wirlung auf das arabische Gummi und den Rohrzucker ausübe.

In einem Zusatze giebt er die Analyse eines Syrups, welchen die Herrn Fouchard zu Neuilly, unter dem Namen Dextrinsyrup fabriciren. Dieser Syrup, welchen sie mittelst gekeimter Gerste oder Kartoffelstärke bereiten, enthält etwa $2 / 5$ seines Gewichts Zucker und etwas mehr als $1 / 5$ gummige Substanz. 